\title{
Atmospheric Muon Neutrino Fraction above 1 GeV
}

\author{
R. Clark, ${ }^{1}$ R. Becker-Szendy, ${ }^{2}$ C. B. Bratton, ${ }^{3}$ J. Breault, ${ }^{4}$ D. Casper, ${ }^{5}$ S. T. Dye, ${ }^{6}$ W. Gajewski, ${ }^{4}$ M. Goldhaber ${ }^{7}$ \\ T. J. Haines,${ }^{8}$ P. G. Halverson, ${ }^{4}$ D. Kielczewska, ${ }^{9}$ W. R. Kropp, ${ }^{4}$ J. G. Learned,${ }^{10}$ J. LoSecco,${ }^{11}$ C. McGrew, ${ }^{12}$ \\ S. Matsuno, ${ }^{10}$ R. S. Miller,${ }^{8}$ L. Price,${ }^{4}$ F. Reines,${ }^{4}$ J. Schultz,${ }^{4}$ H. W. Sobel,${ }^{4}$ J. Stone,${ }^{13}$ \\ L. R. Sulak, ${ }^{13}$ R. Svoboda, ${ }^{1}$ and M. Vagins ${ }^{4}$ \\ ${ }^{1}$ Louisiana State University, Baton Rouge, Louisiana 70803 \\ ${ }^{2}$ Stanford Linear Accelerator Center, Stanford, California 94309 \\ ${ }^{3}$ Cleveland State University, Cleveland, Ohio 44115 \\ ${ }^{4}$ The University of California, Irvine, California 92717 \\ ${ }^{5}$ CERN, Geneva, Switzerland \\ ${ }^{6}$ Hawaii Pacific University, Honolulu, Hawaii, 96813 \\ ${ }^{7}$ Brookhaven National Laboratory, Upton, New York 11973 \\ ${ }^{8}$ Los Alamos National Laboratory, Los Alamos, New Mexico 87544 \\ ${ }^{9}$ Warsaw University, Warsaw, Poland \\ ${ }^{10}$ The University of Hawaii, Honolulu, Hawaii 96822 \\ ${ }^{11}$ The University of Notre Dame, Notre Dame, Indiana 46556 \\ ${ }^{12}$ SUNY at Stoney Brook, Stony Brook, New York 11794 \\ ${ }^{13}$ Boston University, Boston, Massachusetts 02215
}

(Received 12 February 1997)

\begin{abstract}
A 2.1 ktonyr exposure of data from the Irvine-Michigan-Brookhaven detector has yielded 72 atmospheric neutrino events with a vertex contained inside the fiducial volume and at least $0.95 \mathrm{GeV}$ of visible Čerenkov energy. The ratio of these two ratios $\left(\frac{\text { muonlike }}{\text { total }}\right)_{\text {Data }} /\left(\frac{\text { muonlike }}{\text { total }}\right)_{M C}$ was found to be $1.1_{-0.12}^{+0.07}$ (stat) \pm 0.11 (syst). The zenith angle dependence of this ratio of ratios is consistent with being flat. The region of $\sin ^{2}(2 \theta)>0.5$ and $\delta m^{2}>9.8 \times 10^{-3} \mathrm{eV}^{2}$ has been excluded to the $90 \%$ confidence level for $\nu_{\mu} \rightarrow \nu_{e}$ oscillations while the region of $\sin ^{2}(2 \theta)>0.7$ and $\delta m^{2}>1.5 \times 10^{-2} \mathrm{eV}^{2}$ has been excluded to the $90 \%$ confidence level for $\nu_{\mu} \rightarrow \nu_{\tau}$ oscillations. [S0031-9007(97)03604-1]
\end{abstract}

PACS numbers: 14.60.Pq, 96.40.Tv

Cosmic ray interactions in the upper atmosphere produce a large flux of pions and, to a lesser extent, kaons which subsequently produce muon and electron neutrinos. These neutrinos have been observed in underground detectors with the number and distribution of electronlike and muonlike interactions used to search for neutrino oscillations. It is convenient to use a ratio of the neutrino flavors since the absolute flux errors cancel to a large extent. The data can be compared to the Monte Carlo prediction as a ratio of ratios,

$$
f=\frac{\left(\frac{\text { muonlike }}{\text { total }}\right)_{\text {Data }}}{\left(\frac{\text { muonlike }}{\text { total }}\right)_{\mathrm{MC}}},
$$

which will be equal to one if the data agrees with the prediction. The Irvine-Michigan-Brookhaven (IMB) Detector first saw a lower than expected fraction of muonlike events in 1986 [1]. Since then, several experiments have reported values of $f$ below one [2]. More recently, the IMB detector, the Kamiokande detector, and the Soudan 2 detector have reported $f=0.71 \pm 0.04 \pm 0.08$ [3], $f=$ $0.80 \pm 0.04 \pm 0.02[4]$, and $f=0.81_{-0.13}^{+0.07} \pm 0.04[5]$, respectively. In contrast, the Fréjus experiment has reported $f=1.0 \pm 0.06$ [6]. The Kamiokande group has examined their neutrino sample at high energy and reported a zenith angle dependence to $f$ [4]. We present new results in this paper based on a sample of high energy IMB neu- trino events with visible Čerenkov energy above $0.95 \mathrm{GeV}$ and examine the possibility of a zenith angle dependence.

The IMB detector was an 8 kton water Čerenkov detector located in the Morton Salt Mine in Cleveland, Ohio, at a depth of 600 meters, which is 1570 meters of water equivalent. The detector was originally built to look for proton decay and ran from the summer of 1982 to 30 March 1991. It consisted of an $18 \mathrm{~m}$ by $17 \mathrm{~m}$ by $22.5 \mathrm{~m}$ tank of purified water surrounded on all sides by 2048 photomultiplier tubes (PMTs) spaced approximately 1 meter apart. In this analysis, data from IMB-3 is used, which had $20 \mathrm{~cm}$ PMTs with $60 \mathrm{~cm}$ by $60 \mathrm{~cm}$ wave shifter plates attached. Exact details of the construction, calibration, and operation of the detector may be found elsewhere [7].

Originally a cut was applied to all events which had more than 900 PMT hits. This was compatible with the original goal of the detector, which was to observe proton decay, since it removed very high energy events and it kept the amount of archived data to a level feasible for existing storage media. Unfortunately, it also removed the highest energy neutrino events. Higher density data storage devices eventually became available and so the 900 tube cut was removed on 28 February 1990. For this reason, only data collected from then until 30 March 1991, when the detector shut down, is used in this analysis. There were 236 live days in this period. The fiducial volume 
of the detector is defined to be $2.0 \mathrm{~m}$ in from the PMT plane which yields a sensitive mass of $3.3 \mathrm{kton}$, giving a total exposure for the sample of $2.1 \mathrm{kton} y r$. Any event with a vertex inside the fiducial volume is said to be contained even if some or all of the particles exit the detector.

All events were passed through data reduction routines designed to remove events with less than 1000 photoelectrons (PES) of visible Čerenkov energy and events with entering tracks. A total of 22192 events out of roughly $55 \times 10^{6}$ survived these routines. The 22192 events were then visually scanned with a custom graphics display package. This scanning removed events with entering tracks missed by the data reduction routines. Each event was scanned by two different people. To estimate the efficiency of the scanning process, Monte Carlo neutrino events were randomly placed in the sample of real events. From this, the overall efficiency of the visual scanning was calculated to be $0.98 \pm 0.02$ for events with an interaction vertex inside the fiducial volume. The efficiency was $0.98 \pm 0.02$ for $\nu_{e}+\bar{\nu}_{e}$ and $0.99 \pm 0.02$ for $\nu_{\mu}+\bar{\nu}_{\mu}$. The initial scanning left 83 events, 72 of which were fit with a vertex inside the fiducial volume of the detector. Out of these, 41 had multiple tracks while the other 31 had only a single visible track.

Monte Carlo events corresponding to a 29.1 kton yr exposure were generated in the total volume of the detector. Both charged and neutral current interactions were generated for $\bar{\nu}_{e}, \nu_{e}, \bar{\nu}_{\mu}$, and $\nu_{\mu}$ events. Only $17 \%$ of these events had more than 1000 PES of visible Čerenkov energy. The atmospheric neutrino fluxes for the simulation were based on a table by Agrawal et al. [8] extending from 0.8 to $100 \mathrm{GeV}$. As a comparison, the fluxes calculated by Honda et al. [9] were also used, with the results presented in Table I. The median neutrino energy for all the generated events was $1.6 \mathrm{GeV}$. The median neutrino energy for events surviving all cuts was $4.0 \mathrm{GeV}$. For neutrino-nucleon interactions a model was used which included quasielastic scattering and single and multiple pion production. This model successfully reproduces the features of the neutrino events observed in IMB [1]. Tracking of particles through the detector was accomplished with a custom simulation package, except for hadronic interactions which were handled by FLUKA.

The Monte Carlo events, once generated, were treated the same as the data events. They were passed through the

TABLE I. A summary of the data and Monte Carlo events showing the number of electron and muonlike events. The column BGS refers to Monte Carlo events generated with the fluxes from [8], while the HKKM column refers to events generated with the fluxes from [9].

\begin{tabular}{lccc}
\hline \hline & Data & BGS & HKKM \\
\hline Electronlike & 25 & 31.2 & 29.2 \\
Muonlike & 47 & 41.9 & 40.4 \\
Total & 72 & 73.1 & 69.6 \\
\hline \hline
\end{tabular}

same data reduction routines and then visually scanned and hand fitted. Based on this, the efficiency of the whole data reduction process for events inside the fiducial volume and with more than 1000 PES of visible Čerenkov energy was calculated as $0.79 \pm 0.02$. This breaks down as $0.79 \pm 0.05$ for $\bar{\nu}_{e}+\nu_{e}$ charged currents, $0.79 \pm$ 0.03 for $\bar{\nu}_{\mu}+\nu_{\mu}$ charged currents, and $0.80 \pm 0.08$ for neutral currents. $61 \%$ of the Monte Carlo events had multiple tracks, while the rest had only a single visible track. The contribution due to neutral currents in the final Monte Carlo sample is $9 \%$.

Events were identified as being electronlike or muonlike based on a likelihood function $L$. This function was calculated by taking the product of $n$ different factors $P_{i}$, as shown in Eq. (2). Each factor is a ratio of distributions of the measured property $x_{i}$ based on Monte Carlo and cosmic ray events. Some of the properties included were the length of the track, the $d E / d x$ profile, the fraction of light outside the Čerenkov cone, and the probability that a muon decay was observed [10]. Different likelihood functions were used for single and multiple track events since some of the properties were not applicable to multiple track events:

$$
L=\prod_{i=1}^{n} P_{i}\left(x_{i}\right) .
$$

A total of 47 data events were identified as muonlike while 25 were identified as electronlike. In the Monte Carlo sample a total of $57 \%$ of the events were identified as muonlike while the rest were identified as electronlike. The accuracy of the single track likelihood function was calculated as $0.90 \pm 0.03$, while the accuracy of the multiple track likelihood function was calculated as $0.78 \pm 0.04$. The combined accuracy for all events was calculated as $0.85 \pm 0.03$.

A number of systematic errors for the ratio $\left(\bar{\nu}_{\mu}+\nu_{\mu}\right) /$ $\left(\bar{\nu}_{e}+\nu_{e}\right)$ have been estimated for this analysis. The uncertainty due to the atmospheric fluxes in the energy range of this analysis is 5\% [11]. The uncertainty due to the data reduction process based on the Monte Carlo sample was estimated as less than $1 \%$. The uncertainty due to the multiple pion production cross section was estimated as $5 \%$, and the uncertainty due to the flavor misidentification of the events was estimated as $8 \%$. Adding all these errors in quadrature gives a total systematic error of $10 \%$.

Using Eq. (1), the ratio of ratios is

$$
f=1.1_{-0.12}^{+0.07} \text { (stat) } \pm 0.11 \text { (syst) } .
$$

This differs from the previous value of 0.71 obtained by the contained event analysis by IMB [3]. It is worth noting, however, that this value is not inconsistent with the highest momentum $(\geq 1200 \mathrm{MeV} / c)$ events from that analysis which gives a value closer to 0.9. Figure 1 shows the zenith angle distribution of $f$. Also shown are results from Kamiokande [4]. A $\chi^{2}$ test was used to check for 


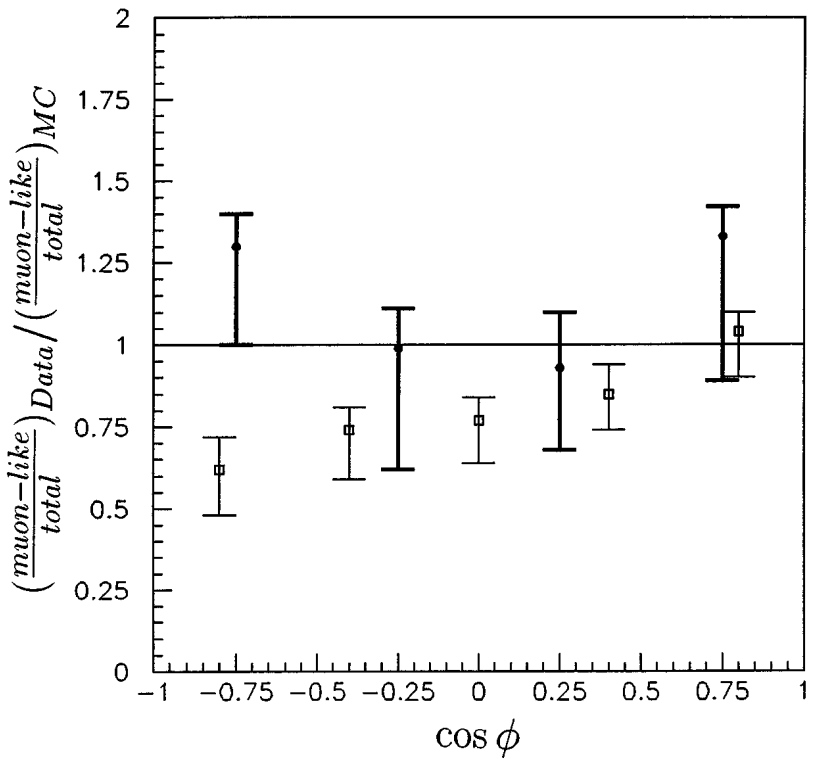

FIG. 1. The zenith angle $(\cos \phi)$ distribution of the ratio of ratios $\left(\frac{\text { muonlike }}{\text { total }}\right)_{\text {Data }} /\left(\frac{\text { muonlike }}{\text { total }}\right)_{\text {MC }}$. The filled circles are from this analysis while the open squares are taken from Kamiokande [4]. Upward going events are on the left side, and the error bars are statistical only.

consistency between the Kamiokande angular distribution and the angular distribution from this analysis. The uncertainties of the two experiments were added in quadrature. These probabilities are based on statistical uncertainties only. The probability that this result is a statistical fluctuation of the Kamiokande angular distribution is 5\%. Based on shape alone, the probability is $23 \%$. Figure 2 shows the zenith angle distribution for electronlike and muonlike events for the data and the Monte Carlo showing the overall agreement between the two.

This result excludes some of the parameter space for neutrino oscillations of $\nu_{\mu} \rightarrow \nu_{x}$. The probability for this oscillation is given as

$$
P_{\nu_{\mu} \rightarrow \nu_{x}}=\sin ^{2}(2 \theta) \sin ^{2}\left(\frac{1.27 \delta m^{2} D}{E_{\nu}}\right),
$$

where $\theta$ is the mixing angle between the states, $\delta m^{2}$ is the difference of the squared neutrino masses, $D$ is the distance from the neutrino creation point $(\mathrm{km})$, and $E_{\nu}$ is the energy of the neutrino $(\mathrm{GeV})$. For underground detectors, the distance $D$ varies from roughly the height of the atmosphere $(10 \mathrm{~km})$ to the diameter of the earth $(13000 \mathrm{~km})$. Our results are not of sufficient accuracy to decide for or against neutrino oscillations but have been used to exclude regions of the parameter spaces for $\nu_{\mu} \rightarrow$ $\nu_{e}$ and $\nu_{\mu} \rightarrow \nu_{\tau}$ to the $90 \%$ confidence level. For $\nu_{\mu} \rightarrow$ $\nu_{e}$ the excluded region is $\sin ^{2}(2 \theta)>0.5$ and $\delta m^{2}>$ $9.8 \times 10^{-3} \mathrm{eV}^{2}$, while for $\nu_{\mu} \rightarrow \nu_{\tau}$ the excluded region is $\sin ^{2}(2 \theta)>0.7$ and $\delta m^{2}>1.5 \times 10^{-2} \mathrm{eV}^{2}$. Figure 3 shows the two excluded regions. The shape of the zenith angular distribution of $f$ was not used in determining these
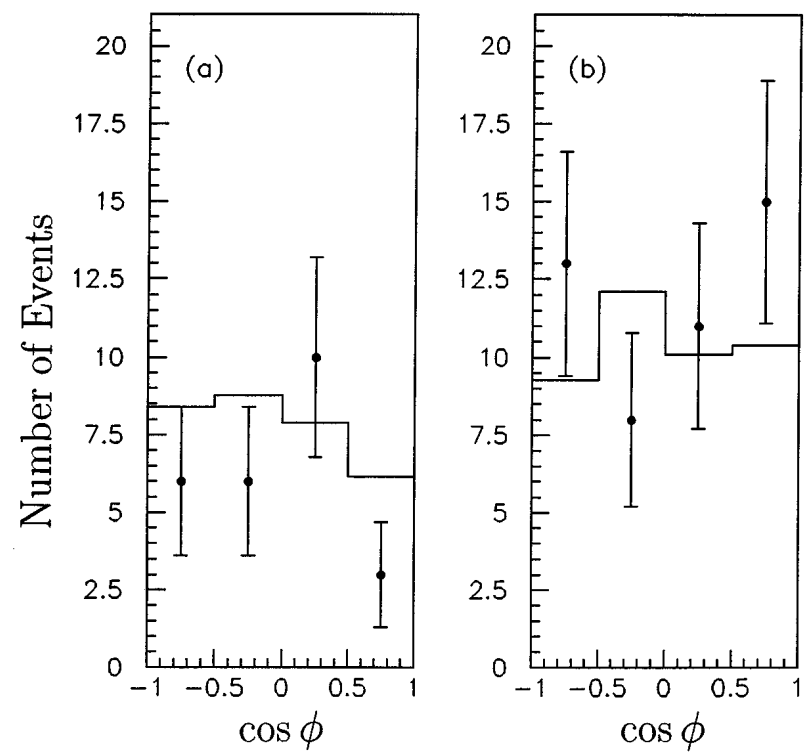

FIG. 2. The zenith angle distribution for electronlike (a) and muonlike (b) events for the data (filled circles) and the Monte Carlo (histogram).

limits. Results from Kamiokande [4] are also shown. It is worthwhile to note that this analysis differs from previous water Čerenkov results in that it does not rely heavily on separating out fully and partially contained events [4], or on rejecting multiple ring events [2].

We wish to thank Morton International, Inc. for hosting this experiment in their Fairport mine, and T. Stanev
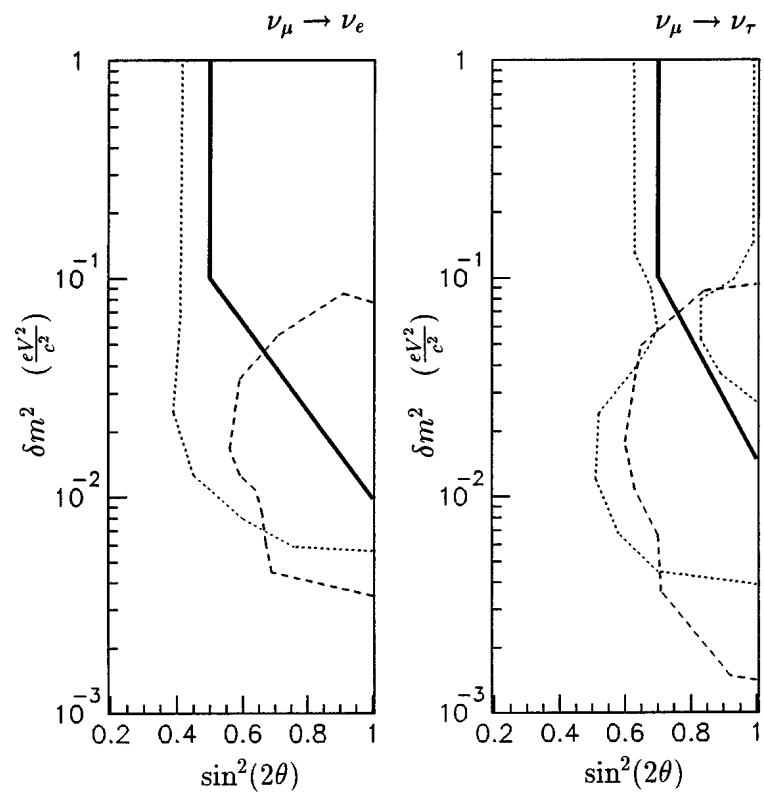

FIG. 3. The parameter space regions for $\nu_{\mu} \rightarrow \nu_{e}$ and $\nu_{\mu} \rightarrow$ $\nu_{\tau}$ oscillations excluded to the $90 \%$ confidence level by this work (solid lines). The allowed regions for the Kamiokande multi-GeV (dashed lines) and sub-GeV (dotted lines) sets [4] are also indicated. The sub-GeV set is similar to earlier IMB sub-GeV results. 
and M. Honda for useful discussions. This work was supported in part by the U.S. Department of Energy, the Los Alamos National Laboratory of the University of California, and the Polish Committee for Scientific Research.

[1] T. J. Haines et al., Phys. Rev. Lett. 57, 1986 (1986).

[2] K.S. Hirata et al., Phys. Lett. B 205, 416 (1988); D. Casper et al., Phys. Rev. Lett. 66, 2561 (1991); K. S. Hirata et al., Phys. Lett. B 280, 146 (1992).

[3] R. Becker-Szendy et al., Phys. Rev. D 46, 3720 (1992).

[4] Y. Fukuda et al., Phys. Lett. B 335, 237 (1994).
[5] M. C. Goodman, Nucl. Phys. B (Proc. Suppl.) 38, 337 (1995).

[6] Ch. Berger et al., Phys. Lett. B 245, 305 (1990).

[7] R. M. Bionta et al., Phys. Rev. Lett. 51, 27 (1983); R. Claus et al., Nucl. Instrum. Methods Phys. Res., Sect. A 261, 540 (1987); R. Becker-Szendy et al., Phys. Rev. D 42, 2974 (1990); R. Becker-Szendy et al., Nucl. Instrum. Methods Phys. Res., Sect. A 352, 629 (1995).

[8] V. Agrawal et al., Phys. Rev. D 53, 1314 (1996).

[9] M. Honda et al., Phys. Rev. D 52, 4985 (1995).

[10] Details of the flavor identification likelihood function may be found in R. Clark, Ph.D. thesis, Louisiana State University, 1997 (unpublished).

[11] T. K. Gaisser et al., Phys. Rev. D 54, 5578 (1996). 